Ass.

FORSCHUNG - AUSBILDUNG - WEITERBILDUNG

Bericht Nr. 84

ON A TRANSMISSION BOUNDARY-VALUE PROBLEM FOR THE TIME-HARMONIC MAXWELL EQUATIONS WITHQUT DISPLACEMENT CURRENTS<smiles>CCC(C)(C)CC[As]</smiles>

UNIVERSITÄT KAISERSLAUTERN

Fachbereich Mathematik

Postfach 3049

W-6750 Kaiserslautern

Dezember 1992 
MAT $144 \mid 620-84$

$93 \mathrm{~g} 540 \mathrm{c1}$ 


\title{
On a Transmission Boundary - Value Problem for the Time - Harmonic Maxwell Equations without Displacement Currents
}

\author{
Martin Reissel \\ Fachbereich Mathematik \\ Universitaet Kaiserslautern \\ D - 6750 Kaiserslautern \\ Germany
}

\begin{abstract}
We consider a transmission boundary-value problem for the lime-harmonic Maxwell equations neglecting displacement currents. The usual transmission conditions. which require the continuity of the tangential components of the electric and magnetic fields across boundaries are slightly modified. For this new problem we show that the uniqueness of the solution depends on the lopological properties of the domains under considerartion. Finally we obtain existence results by using a boundary integral equation approach.
\end{abstract}

\section{Introduction}

Many problems in electrical engineering lead to transmission boundary - value problems for the time harmonic Maxwell equations. A standard problem of this type is shown in Fig. 1. One considers a bounded domain $G^{E} \subset \mathbb{R}^{3}$ of conducting material which is surrounded by an isolator (usually air). In the interior of the unbounded domain $G^{L}:=\mathbb{R}^{3} \backslash \bar{G}^{E}$ a time - harmonic current density $\tilde{J}_{e}(x, t)=J_{e}(x) e^{-i \omega t}$ is given. We are now interested in the currents induced in $G^{E}$ by $\tilde{J}_{e}$. This leads to the classical transmission boundary - value problem for the time harmonic Maxwell equations

$$
\begin{array}{lll}
\operatorname{curl} H^{L}=J_{e}-i \omega \varepsilon^{L} E^{L} & \text { in } G^{L}, & \operatorname{curl~} H^{E}=\left(\sigma^{E}-i \omega \varepsilon\right. \\
\operatorname{curl} E^{L}=i \omega \mu^{L} H^{L} & & \operatorname{curl} E^{E}=i \omega \mu^{E} H^{E} \\
& n \wedge H^{L}=n \wedge H^{E} & \text { on } \partial G^{E}, \\
& n \wedge E^{L}=n \wedge E^{E}
\end{array}
$$


with Silver-Müller radiation condition

$$
H^{L} \wedge \frac{x}{|x|}-E^{L}=o\left(\frac{1}{|x|}\right)
$$

uniformly for $|x| \rightarrow \infty$.

The different constants have the following meaning:

$$
\begin{array}{ll}
\omega \geq 0 & \text { frequency, } \\
\varepsilon^{L}, \varepsilon^{E}>0 & \text { electric permittivity in } G^{L}, G^{E}, \\
\mu^{L}, \mu^{E}>0 & \text { magnetic permeability in } G^{L}, G^{E}, \\
\sigma^{E}>0 & \text { electric conductivity in } G^{E} .
\end{array}
$$

Under certain assumptions on the regularity of $\mathrm{J}_{\mathrm{e}}$ and the smoothness of the boundary $\Gamma$, which separates the domains $G^{L}$ and $G^{E}$, existence and uniqueness of solutions $\mathrm{H}^{\mathrm{L}}, \mathrm{E}^{\mathrm{L}}, \mathrm{H}^{\mathrm{E}}, \mathrm{E}^{\mathrm{E}}$ of (1) can be shown [7,9].

Dealing with problems in connection with machines working at power frequencies, equations (1) are modified. Since the frequency $\omega$ is very small, displacement currents are usually neglected, which means that $\varepsilon^{L}$ and $\varepsilon^{E}$ are set to 0 in (1): Moreover the transmission and radiation conditions are changed. The continuity of the tangential components of the electric field across $\Gamma$ is substituted by the condition $n \cdot\left(\mu_{0} H^{L}\right)=n \cdot\left(\mu H^{E}\right)$ on $\Gamma$, $n$ being the outer normal to $G^{E}$. In addition the Silver-Muiller radiation condition is replaced by $H^{L}(x)=o(1), E^{L}(x)=o(1)$ uniformly for $|\mathbf{x}| \rightarrow \infty$. All these modifications together yield our new problem:

$$
\begin{aligned}
& \begin{array}{lll}
\operatorname{curl} \mathrm{H}^{\mathrm{L}}=\mathrm{J}_{e} & \operatorname{curl} \mathrm{H}^{\mathrm{E}}=\sigma^{E} \mathrm{E}^{\mathrm{E}} \\
\operatorname{curl} \mathrm{E}^{\mathrm{L}}=\mathrm{i} \omega \mu^{\mathrm{L}} \mathrm{H}^{\mathrm{L}} & \text { in } \mathrm{G}^{\mathrm{L}}, & \operatorname{curl} \mathrm{E}^{\mathrm{E}}=i \omega \mu^{E} \mathrm{II}^{E}
\end{array} \quad \text { in } \mathrm{G}^{\mathrm{E}}, \\
& \begin{array}{c}
n \wedge H^{E}=n \wedge H^{L} \\
n \cdot\left(\mu^{E} H^{E}\right)=n \cdot\left(\mu^{L} H^{L}\right)
\end{array} \quad \text { on } \Gamma \\
& H^{L}(x)=o(1), \quad E^{L}(x)=o(1) \quad \text { uniformly for }|x| \rightarrow \infty \text {. }
\end{aligned}
$$

As we will see, uniqueness results for (2) will strongly depend on the topology of $G^{L}$ resp. $G^{E}$. In chapter 4 , we will show existence for $(2)$ and the set of all possible solutions will be completely characterized. 


\section{Preliminaries}

Before we start with the existence and uniqueness proof, we want to give a detailed description of the problem.

Let $C(G)\left(C^{k}(G)\right.$ resp. $\left.C^{0 \alpha}(G), 0<\alpha<1\right)$ denote the space of continuous ( $k$ times continuously differentiable resp. hölder - continuous) functions on $G$.

$G^{E} \subset \mathbb{R}^{3}$ is an open, bounded domain with $C^{\dot{2}}$ boundary. The complement $G^{L}=\mathbb{R}^{3} \backslash \bar{G}^{E}$ should be connected $\left(\bar{G}^{E}\right.$ denotes the closure of $\left.G^{E}\right)$. $G^{E}$ is the union of $m$ connected components $G_{j}^{E}, j=1, \ldots, m$ having the topological genus $p_{j}$. The boundaries $\Gamma_{j}=\partial G_{j}^{E}$ are closed surfaces, which should be disjoint. Setting $\Gamma=\bigcup_{j=1}^{m} \Gamma_{j}$ we get $\Gamma=\partial G^{E}=\partial G^{L}$.

The topological genus of $G^{E}$ resp. $G^{L}$ is $p=\sum_{j=1}^{m} p_{j}$. There exist $p$ surfaces $\Sigma_{i}^{E} \subset G^{E}$ resp. $\quad \Sigma_{i}^{L} \subset G^{L}, \quad i=1, \ldots, p$, such that $G^{E} \backslash \bigcup_{i=1}^{p} \Sigma_{i}^{E} \quad$ resp. $G^{L} \backslash \bigcup_{i=1}^{p} \Sigma_{i}^{L}$ are simply connected. The boundary curves $\gamma_{i}^{L}=\partial \Sigma_{i}^{E}$ and $\gamma_{i}^{E}=\partial \Sigma_{i}^{L}$ lie on $\Gamma$.

\section{Example}

Let $G^{E}$ be a torus. In this case we have $m=p=1$. The surfaces $\Sigma_{1}^{E}, \Sigma_{1}^{L}$ and the curves $\gamma_{1}^{L}, \gamma_{1}^{E}$ are shown in Fig. 2.

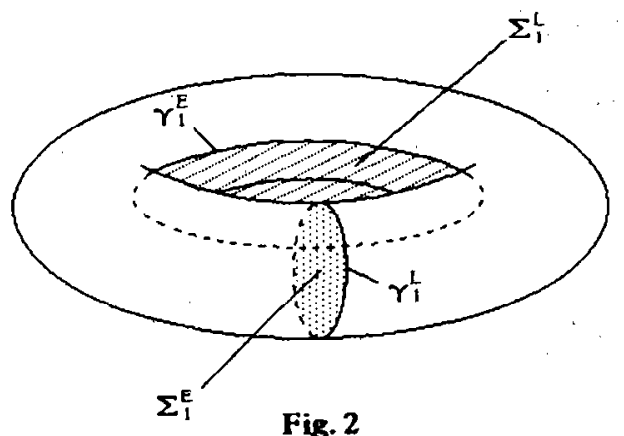

Fig. 2

The problem to be solved, is now defined as :

For $J_{e} \in C^{l}\left(\mathbb{R}^{3}\right), \quad \operatorname{div} J_{e}=0, \quad \operatorname{supp}\left(J_{e}\right) \subset G^{J}, \quad \bar{G}^{J} \subset G^{L}$ bounded,

find $H^{L}, E^{L} \in C^{1}\left(G^{L}\right) \cap C\left(\bar{G}^{L}\right), \quad H^{E}, E^{E} \in C^{1}\left(G^{E}\right) \cap C\left(\bar{G}^{E}\right)$ solving

$$
\begin{array}{lll}
\operatorname{curl} H^{L}=J_{e} & \text { in } G^{L}, & \operatorname{curl} H^{E}=\sigma^{E} E^{E} \\
\operatorname{curl} E^{L}=i \omega \mu^{L} H^{L} & & \operatorname{curl} E^{E}=i \omega \mu^{E} H^{E}
\end{array} \quad \text { in } G^{E},
$$$$
\begin{aligned}
n \wedge H^{E} & =n \wedge H^{L} \\
n \cdot\left(\mu^{E} H^{E}\right) & =n \cdot\left(\mu^{L} H^{L}\right)
\end{aligned} \quad \text { on } \Gamma,
$$

$$
H^{L}(x)=o(1), \quad E^{L}(x)=o(1) \quad \text { uniformly for }|x| \rightarrow \infty \text {. }
$$




\section{Uniqueness}

Taking a closer look at (3),(4),(5), it becomes obvious, that we can not expect uniqueness for all four fields, because adding the gradient of a suitably chosen function to $E^{\mathrm{L}}$ does not change anything in (3),(4),(5). Therefore, if we talk about uniqueness in the sequel, we only mean uniqueness of the fields $\mathrm{H}^{\mathrm{L}}, \mathrm{H}^{\mathrm{E}}$ and $\mathrm{E}^{\mathrm{E}}$.

\section{Theorem 1}

For problem $(3),(4),(5)$ together with the additional condition

$$
\int_{\gamma_{i}^{L}} \tau \cdot H^{L} d l=h_{i}^{L}, \quad i=1, \ldots, p,
$$

$h_{i}^{L} \in \mathbb{C}$ given, $\tau$ being the unit tangent to $\gamma_{i}^{L}$, the fields $H^{L}, H^{E}, E^{E}$ are uniquely determined.

\section{Proof}

We consider the homogeneous problem with $J_{e}=0$ and $h_{i}^{L}=0, i=1, \ldots, p$. We show that the fields $\mathrm{H}^{\mathrm{L}}, \mathrm{H}^{\mathrm{E}}, \mathrm{E}^{\mathrm{E}}$ vanish identically.

From the first transmission condition $n \wedge H^{E}=n \wedge H^{L}$ on $\Gamma$, we get, with the help of the Gaussian theorem

$$
\int_{\Gamma} n \cdot\left(\bar{H}^{L} \wedge E^{E}\right) d s=\int_{\Gamma} n \cdot\left(\bar{H}^{E} \wedge E^{E}\right) d s=\int_{G}\left(\sigma^{E} E^{E} \cdot \bar{E}^{E}-i \omega \mu^{E} H^{E} \cdot \bar{H}^{E}\right) d v
$$

where $\bar{F}$ denotes the complex conjugate of the field $F$.

$\mathrm{G}^{\mathrm{E}}$ and $\mathrm{G}^{\mathrm{L}}$ were defined to have topological genus $\mathrm{p}$. In this case, it is well known [6], that there exist $p$ linear independent Neumann fields $Z_{i}^{E}$ resp. $Z_{i}^{L}$, $\mathrm{i}=1, \ldots, p$, in $\mathrm{G}^{\mathrm{E}}$ resp. $\mathrm{G}^{\mathrm{L}}$, fulfilling

$$
\begin{aligned}
& \operatorname{curl} Z_{i}^{E}=0, \quad \operatorname{div} Z_{i}^{E}=0 \quad \text { in } G^{E}, \quad n \cdot Z_{i}^{E}=0 \quad \text { on } \Gamma \text {; } \\
& \operatorname{curl} Z_{i}^{L}=0, \quad \operatorname{div} Z_{i}^{L}=0 . \quad \text { in } G^{L}, \quad n \cdot Z_{i}^{L}=0, \quad \text { on } \Gamma \text {, } \\
& \int_{\gamma_{i}^{L}} \tau \cdot Z_{j}^{L} d l=\delta_{i j}, \quad \int_{\gamma_{i}^{E}} \tau \cdot Z_{j}^{L} d l=0, \quad \int_{\gamma_{i}^{E}} \tau \cdot Z_{j}^{E} d l=\delta_{i j}, \quad \int_{\gamma_{i}^{L}} \tau \cdot Z_{j}^{E} d l=0
\end{aligned}
$$

and

$$
Z_{i}^{L}(x)=O\left(\frac{1}{|x|^{2}}\right)
$$

uniformly for $|x| \rightarrow \infty$. As a consequence of the regularity assumptions on $\mathrm{G}^{\mathrm{E}}$ and $G^{L}$ we get

$$
Z_{i}^{E} \in C^{\infty}\left(G^{E}\right) \cap C^{0 \alpha}\left(\bar{G}^{E}\right), \quad Z_{i}^{L} \in C^{\infty}\left(G^{L}\right) \cap C^{0 \alpha}\left(\bar{G}^{L}\right) .
$$


Using the second transmission condition $n \cdot\left(\mu^{\mathrm{L}} \cdot \mathrm{H}^{\mathrm{L}}\right)=\mathrm{n} \cdot\left(\mu^{\mathrm{E}} \mathrm{H}^{\mathrm{E}}\right)$ on $\Gamma$, we conclude that for any surface element $S \subset \Gamma$ we have

$$
\int_{\partial S}^{\prime} \tau \cdot\left(E^{E}-E^{L}\right) d l=\int_{S} n \cdot \operatorname{curl}\left(E^{E}-E^{L}\right) d s=i \omega \int_{S} n \cdot\left(\mu^{E} H^{E}-\mu^{L} H^{L}\right) d s=0
$$

But this means, that the tangential components of $E^{E}-E^{L}$ on $\Gamma$ are of the form

$$
\left.\left(E^{E}-E^{L}\right)\right|_{\tan }=\operatorname{Grad} \varphi+\sum_{j=1}^{p} e_{j}^{L} Z_{j}^{L}+\sum_{j=1}^{p} e_{j}^{E} Z_{j}^{E} \text {, }
$$

where $\operatorname{Grad} \varphi$ denotes the surface gradient of $\varphi$ on $\Gamma$ and $e_{i}^{L}, e_{i}^{E} \in \mathbb{C}, i=1, \ldots, p$, are complex numbers.

In complete analogy, we derive from

$$
\operatorname{curl} \mathrm{H}^{\mathrm{L}}=0 \quad \text { in } \mathrm{G}^{\mathrm{L}}, \quad \quad \int_{\gamma_{\mathrm{i}}^{\mathrm{L}}} \tau \cdot \mathrm{H}^{\mathrm{L}} \mathrm{dl}=0, \quad \mathrm{i}=1, \ldots, \mathrm{p} \text {, }
$$

that we can write the tangential components of $\mathrm{H}^{\mathrm{L}}$ as a surface gradient

$$
\left.H^{L}\right|_{\tan }=\operatorname{Grad} \psi \text {. }
$$

Putting $\left.\left(E^{E}-E^{L}\right)\right|_{\tan }$ and $H^{L} / \tan$ in $(7)$, we arrive at

$$
\begin{aligned}
\int_{\Gamma} n \cdot\left(\bar{H}^{E} \wedge E^{E}\right) d s & =\int_{\Gamma} n \cdot\left(\bar{H}^{L} \wedge E^{E}\right) d s \\
& =\int_{\Gamma} n \cdot\left(\operatorname{Grad} \bar{\psi} \wedge\left(E^{L}+\operatorname{Grad} \varphi+\sum_{i=1}^{p} e_{i}^{L} Z_{i}^{L}+\sum_{i=1}^{p} e_{i}^{E} Z_{i}^{E}\right)\right) d s .
\end{aligned}
$$

Applying Stokes' theorem to the terms on the right hand side, we deduce

$$
\begin{aligned}
& \int_{\Gamma} n \cdot(\operatorname{Grad} \bar{\psi} \wedge \operatorname{Grad} \varphi) d s=0, \\
& \int_{\Gamma} n \cdot\left(\operatorname{Grad} \bar{\psi} \wedge Z_{i}^{L}\right) d s=0, \\
& \int_{\Gamma} n \cdot\left(\operatorname{Grad} \bar{\psi} \wedge Z_{i}^{E}\right) d s=0
\end{aligned}
$$

and therefore

$$
\int_{\Gamma} n \cdot\left(\bar{H}^{E} \wedge E^{E}\right) d s=\int_{\Gamma} n \cdot\left(\bar{H}^{L} \wedge E^{L}\right) d s .
$$

Let us now consider $G^{R}=G^{L} \cap B^{R}, B^{R}:=\left\{x\left|x \in \mathbb{R}^{3},\right| x \mid \leq R\right\}$. For large enough $R$, we get by using the Gaussian theorem

$$
\int_{\partial B^{R}} \frac{x}{|x|} \cdot\left(\bar{H}^{L} \wedge E^{L}\right) d s-\int_{\Gamma} n \cdot\left(\bar{H}^{L} \wedge E^{L}\right) d s=\int_{\partial G^{R}} n^{\prime} \cdot\left(\bar{H}^{L} \wedge E^{L}\right) d s=-i \omega \mu^{L} \int_{G^{R}} H^{L} \cdot \bar{H}^{L} d v,
$$

$n$ ' being the outer normal to $G^{R}$. 
Together with (7) and (8) this means

$$
-i \omega \mu^{L} \int_{G^{R}} H^{L} \cdot \bar{H}^{L} d s+\int_{G^{E}}\left(\sigma^{E} E^{E} \cdot \bar{E}^{E}-i \omega \mu^{E} H^{E} \cdot \bar{H}^{E}\right) d v=\int_{\partial B^{R}} \frac{x}{|x|} \cdot\left(\bar{H}^{L} \wedge E^{L}\right) d s .
$$

For $\mathrm{H}^{\mathrm{L}}$ we have

$$
\operatorname{curl} H^{L}=0, \quad \operatorname{div} H^{L}=0 \quad \text { in } G^{L}, \quad H^{L}(x)=o(1) \text { uniformly for }|x| \rightarrow \infty .
$$

Therefore $H^{L}$ is a harmonic vectorficld in $G^{L}$ tending to 0 for $|x| \rightarrow \infty$ and thus [6]

$$
H^{L}(x)=O\left(\frac{1}{|x|^{2}}\right), \quad \text { uniformly for }|x| \rightarrow \infty .
$$

From $E^{L}(x)=o(1)$ uniformly for $|x| \rightarrow \infty$ we get

$$
\left|\frac{x}{|x|} \cdot\left(\bar{H}^{L} \wedge E^{L}\right)\right|=o\left(\frac{1}{R^{2}}\right) \quad \text { on } \partial B^{R}, \quad R \rightarrow \infty
$$

and

$$
\int_{\partial B^{R}} \mid \frac{x}{|x|} \cdot\left(\bar{H}^{L} \wedge E^{L}\right) d s=o(1) \quad \text { for } R \rightarrow \infty .
$$

Taking the limit $R \rightarrow \infty$ equation (9) yields

$$
\sigma^{E} \int_{G^{E}} E^{E} \cdot \bar{E}^{E} d v-i \omega\left(\mu^{E} \int_{G^{E}} H^{E} \cdot \bar{H}^{E} d v+\mu^{L} \int_{G^{L}} H^{L} \cdot \bar{H}^{L} d v\right)=0 .
$$

Since $\omega, \sigma^{\mathrm{E}}, \mu^{\mathrm{L}}, \mu^{\mathrm{E}}$ are real and positive constants, we conclude

$$
\mathrm{H}^{\mathrm{L}} \equiv 0, \quad \mathrm{E}^{\mathrm{E}} \equiv 0, \quad \mathrm{H}^{\mathrm{E}} \equiv 0 \text {. }
$$

\section{Remark}

The free parameters $h_{i}^{L}$ in (6), which are the circulations of $H^{L}$ along the curves $\gamma_{i}^{L}$, are later on used to characterize the different solutions of $(3),(4),(5)$. 


\section{Existence}

To establish existence results for $(3),(4),(5)$, we consider the following auxiliary problem :

Find $H^{L} \in C^{l}\left(G^{L}\right) \cap C\left(\bar{G}^{L}\right)$,

$$
H^{E} \in C^{2}\left(G^{E}\right) \cap C\left(\bar{G}^{E}\right), \quad \operatorname{div} H^{E} \in C\left(\bar{G}^{E}\right), \quad \operatorname{curl} H^{E} \in C\left(\bar{G}^{E}\right)
$$

solving

$$
\begin{array}{rlrl}
\operatorname{curl} H^{L}=0 & \left(\Delta+k^{2}\right) H^{E}=0 \\
\operatorname{div} H^{L}=0 & \text { in } G^{L}, & k^{2}=i \omega \sigma^{E} \mu^{E}
\end{array} \quad \text { in } G^{E},
$$

$$
\begin{array}{ll}
n \wedge H^{E}-n \wedge H^{L}=c & \text { on } \Gamma, \\
n \cdot\left(\mu^{E} H^{E}\right)-n \cdot\left(\mu^{L} H^{L}\right)=g & i=1, \ldots, p, \\
\operatorname{div~} H^{E}=d & \text { uniformly for }|x| \rightarrow \infty . \\
\int_{\gamma_{i}^{L} \tau} \tau H^{L} d l=0 &
\end{array}
$$

For $\mathrm{k}$ we choose the square root with positive imaginary part.

In this chapter, we show, that $(10)$ is uniquely solvable for sufficiently smooth data $\mathrm{c}, \mathrm{g}, \mathrm{d}$. Moreover we describe, how all solutions of $(3),(4),(5)$ can be constructed by using the solvability of the auxiliary problem (10).

By the following Lemma [8], the uniqueness of $(10)$ can be reduced to the uniqueness theorem given in the last chapter.

\section{Lemma 1}

Let $H \in C^{1}\left(G^{L}\right) \cap C\left(\bar{G}^{L}\right), H(x)=O\left(\frac{1}{|x|^{1+\beta}}\right),|x| \rightarrow \infty, O<\beta<2$, satisfying $\operatorname{div} H=0$ in $G^{L}$. If we have

$$
\int_{\Gamma_{j}} n \cdot H d s=0, \quad j=1, \ldots, m
$$

for any connected component $\Gamma_{j}$ of $\Gamma$, there exists a field Field $E \in C^{1}\left(G^{L}\right) \cap C\left(\overleftarrow{G}^{L}\right)$, such that

$$
\begin{array}{ll}
\operatorname{curl} E=i \omega \mu^{L} H, & \operatorname{div} E=0 \quad \text { in } G^{L}, \\
E(x)=O\left(\frac{1}{|x|^{\beta}}\right), & \text { uniformly for } \quad|x| \rightarrow \infty .
\end{array}
$$




\section{Theorem 2}

Problem (10) has at most one solution.

\section{Proof}

We consider $(10)$ with homogeneous data $c=0, g=0, d=0$. Since $\mathrm{H}^{\mathrm{E}}$ is a solution of the vector Helmholtz equation with wave number $k$ in $\mathrm{G}^{\mathrm{E}}$, the divergence of $\mathrm{H}^{\mathrm{E}}$ solves the scalar Helmholtz equation with the same wave number $\mathrm{k}$ in $\mathrm{G}^{\mathrm{E}}$. From $\left.\operatorname{divH}\right|_{\Gamma}=d=0$ and $\operatorname{Im}(k)>0$ it follows, that $\operatorname{div} \mathrm{H}^{\mathrm{E}}$ vanishes identically in $G^{r}[1]$. Using the identity

$$
\text { curl curl }=\operatorname{grad} \operatorname{div}-\Delta
$$

and defining $E^{E}$ by

$$
\mathrm{E}^{\mathrm{E}}=\frac{1}{a^{\mathrm{E}}} \operatorname{curl} \mathrm{H}^{\mathrm{E}}
$$

we conclude, that $\mathrm{H}^{\mathrm{E}}, \mathrm{E}^{\mathrm{E}}$ are solutions of the Maxwell equations in $\mathrm{G}^{\mathrm{E}}$.

On the other hand, $H^{L}$ is a harmonic vector field in $G^{L}$, satisfying $H^{L}(x)=o(1)$ uniformly for $|x| \rightarrow \infty$. Therefore, we immediately get $H^{L}(x)=O\left(\frac{1}{|x|^{2}}\right)$ uniformly for $|x| \rightarrow \infty$. In addition to this, we deduce from the transmission conditions of (10)

$$
\begin{aligned}
\mu^{L} \int_{\Gamma_{j}} n \cdot H^{L} d s & =\int_{\Gamma_{j}} n \cdot\left(\mu^{L} H^{L}\right) d s=\int_{\Gamma_{j}} n \cdot\left(\mu^{E} H^{E}\right) d s \\
& =\frac{1}{i \omega} \int_{\Gamma_{j}} n \cdot\left(i \omega \mu^{E} H^{E}\right) d s=\frac{1}{i \omega} \int_{\Gamma_{j}} n \cdot \operatorname{curl} E^{E} d s=0 .
\end{aligned}
$$

Applying Lemma 1 with $\beta=2$ to $H^{L}$ proves the existence of a field $E^{L}$ defined in $\mathrm{G}^{\mathrm{L}}$, having the following properties :

$$
\begin{aligned}
& \text {. } \operatorname{curl} E^{L}=i \omega \mu^{L} H^{L} \quad \text { in } G^{L}, \\
& E^{L}(x)=o(1) \quad \text { uniformly for }|x| \rightarrow \infty .
\end{aligned}
$$

Thus we have shown that $H^{L}, E^{L}, H^{E}, E^{E}$ solve the homogeneous equations (3), (4), (5) with vanishing circulations

$$
\int_{\gamma_{i}^{L}} \tau \cdot H^{L} \cdot d l=0 \text {. }
$$

Now, from Theorem 1 we conclude $\mathrm{H}^{\mathrm{L}} \equiv 0, \mathrm{H}^{\mathrm{E}} \equiv 0$.

Before we start with the existence proof for $(10)$, we have to introduce some notation. 


\section{Definition}

Let $0<\alpha<1$

$-C^{0 \alpha}(G), \quad\|u\|_{0 \alpha, G}=\sup _{x \in G}|u(x)|+\sup _{\substack{x \rightarrow y \\ x, y \in G}} \frac{|u(x)-u(y)|}{|x-y|^{\alpha}}$,

is the space of Hölder continuous functions on $G$.

- $\mathrm{V}^{0 \alpha}(\Gamma)=\left(C^{0 \alpha}(\Gamma)\right)^{3}, \quad\|\mathrm{a}\|_{\mathrm{V} \alpha . \Gamma}=\max _{i=1.2 .3}\left(\left\|\mathrm{a}_{\mathrm{i}}\right\|_{0 \alpha . \Gamma}\right)$,

is the space of Hölder continuous vector fields on $\Gamma$.

- $\mathrm{T}^{0 \alpha}(\Gamma)=\left\{\mathrm{a} \in \mathrm{V}^{0 \alpha}(\Gamma) \mid \mathrm{n} \cdot \mathrm{a}=0\right\}, \quad\|\mathrm{a}\|_{\mathrm{T} \alpha, \Gamma}=\|\mathrm{a}\|_{\mathrm{V} \alpha, \Gamma}$,

is the space of Hölder continuous tangential fields on $\Gamma$.

$-T_{d}^{0 \alpha}(\Gamma)=\left\{a \in T^{0 \alpha}(\Gamma) \mid \operatorname{Div} a \in C^{0 \alpha}(\Gamma)\right\}, \quad\|u\|_{d \alpha, \Gamma}=\max \left(\|u\|_{T \alpha, \Gamma},\|\operatorname{Div} u\|_{0 \alpha, \Gamma}\right)$,

is the space of Hölder continuous tangential fields on $\Gamma$ having Hölder continuous surface divergence.

- $X_{d}^{0 \alpha}(\Gamma)=T_{d}^{0 \alpha}(\Gamma) \times C^{0 \alpha}(\Gamma) \times C^{0 \alpha}(\Gamma)$,

$\|\mathrm{u}\|_{\mathrm{X}_{\mathrm{d}} \alpha}=\max \left(\left\|\mathrm{u}_{1}\right\|_{d \alpha, \Gamma},\left\|\mathrm{u}_{2}\right\|_{0 \alpha, \Gamma},\left\|\mathrm{u}_{3}\right\|_{0 \alpha, \Gamma}\right)$.

$-\Phi(x, y)=\frac{1}{4 \pi} \frac{e^{i k|x-y|}}{|x-y|}, \quad \Phi_{0}(x, y)=\frac{1}{4 \pi} \frac{1}{|x-y|}$.

The spaces $C^{0 \alpha}(G), V^{0 \alpha}(\Gamma), T^{0 \alpha}(\Gamma), T_{d}^{0 \alpha}(\Gamma)$ and $X_{d}^{0 \alpha}$ equipped with the corresponding norms are Banach spaces.'

\section{Theorem 3}

For any $c \in T_{d}^{0 \alpha}(\Gamma), g \in C^{0 \alpha}(\Gamma), d \in C^{0 \alpha}(\Gamma)$, problem (10) is uniquely solvable. The solution depends continuously on the given data.

\section{Proof}

The proof will be divided into three parts. In the first part, we use a special ansatz for $\mathrm{H}^{\mathrm{L}}$ and $\mathrm{H}^{\mathrm{E}}$ to transform the transmission boundary-value problem into a boundary integral equation. In the second step, we show that the integral equation is of second kind. Finally we conclude the proof by showing the injectivity of the corresponding integral operator. 


\section{The Ansatz}

We are looking for solutions $\mathrm{H}^{\mathrm{L}}, \mathrm{H}^{\mathrm{E}}$ of the form

$$
\begin{aligned}
H^{L}(x)=\operatorname{grad}_{x} \int_{\Gamma} \lambda(y) \Phi_{0}(x, y) d s(y) & \\
H^{E}(x)=\operatorname{curl}_{x} \int_{\Gamma} a(y) \Phi(x, y) d s(y) & +\operatorname{grad}_{x} \int_{\Gamma} \lambda(y) \Phi(x, y) d s(y) \\
& +\int_{\Gamma} n(y) \delta(y) \Phi(x, y) d s(y)
\end{aligned}
$$

$a \in T_{d}^{0 \alpha}(\Gamma), \lambda \in C^{0 \alpha}(\Gamma), \delta \in C^{0 \alpha}(\Gamma)$. For $H^{L}, H^{E}$ we get $[1,9]$

(i) $\quad H^{L} \in C^{\infty}\left(G^{L}\right) \cap C^{0 \alpha}\left(\bar{G}^{E}\right), \quad H^{L}(x)=O\left(\frac{1}{|x|^{2}}\right)$, uniformly for $|x| \rightarrow \infty$,

$$
\int_{\gamma_{i}^{L}} \tau \cdot H^{L} d l=0, \quad i=1, \ldots, p .
$$

(ii) $H^{E} \in C^{2}\left(G^{E}\right) \cap C^{0 \alpha}\left(\bar{G}^{E}\right), \quad \operatorname{div} H^{E} \in C^{0 \alpha}\left(\bar{G}^{E}\right), \quad \operatorname{curl} H^{E} \in C^{0 \alpha}\left(\bar{G}^{E}\right)$.

(iii) $\operatorname{curl} \mathrm{H}^{\mathrm{L}}=0$

$$
\begin{array}{lll}
\operatorname{curl} H^{L}=0 & \left(\Delta+k^{2}\right) H^{E}=0 \\
\operatorname{div} H^{L}=0 & k^{2}=i \omega \sigma^{E}{ }^{E}
\end{array}
$$

(iv) $\left\|H^{\mathrm{L}}\right\|_{\mathrm{V} \alpha, \bar{G}^{\mathrm{L}}} \leq \mathrm{c}_{\alpha}\|\lambda\|_{0 \alpha, \Gamma}$,

$$
\begin{aligned}
& \max \left(\left\|H^{E}\right\|_{V \alpha, \bar{G}^{E}},\left\|\operatorname{div} H^{E}\right\|_{0 \alpha, \bar{G}^{E}}\right) \leq c_{\alpha}\left\|\left(\begin{array}{l}
\lambda \\
\delta
\end{array}\right)\right\|_{X_{d} \alpha}, \\
& \left\|\operatorname{curl} H^{E}\right\|_{V \alpha, \bar{G}^{E}} \leq c_{\alpha} \max \left(\|a\|_{d \alpha, \Gamma},\|\delta\|_{0 \alpha, \Gamma}\right) .
\end{aligned}
$$

From (i),...,(iv) we see, that our ansatz meets all the regularity requirements of $(10)$. $H^{L}$ and $H^{E}$ depend continuously on $a, \lambda$ and $\delta$ and solve the required differential equations. Therefore we only have to adjust the boundary values on $\Gamma$ corresponding to $(10)$.

Defining $F_{ \pm}(x)=\lim _{h \times 0} F(x \pm h n(x)), x \in \Gamma, n$ outer normal to $G^{E}$, we get the following jump conditions for single and double layer potentials and their derivatives [1]:

$$
\begin{aligned}
\left.\operatorname{curl}_{x} \int_{\Gamma} a(y) \Phi(x, y) d s(y)\right|_{ \pm} & =\int_{\Gamma} \operatorname{curl}(a(y) \Phi(x, y)) d s(y) \mp \frac{1}{2} n(x) \wedge a(x), \\
\left.\operatorname{grad}_{x} \int_{\Gamma} \lambda(y) \Phi(x, y) d s(y)\right|_{:} & =\int_{\Gamma} \lambda(y) \operatorname{grad}_{x} \Phi(x, y) d s(y) \mp \frac{1}{2} n(x) \lambda(x), \\
\left.\int_{\Gamma} n(y) \delta(y) \Phi(x, y) d s(y)\right|_{:} & =\int_{\Gamma} n(y) \delta(y) \Phi(x, y) d s(y), \\
\left.\int_{\Gamma} \lambda(y) \Phi(x, y) d s(y)\right|_{ \pm} & =\int_{\Gamma} \lambda(y) \Phi(x ; y) d s(y), \\
\left.\int_{\Gamma} \delta(y) \partial_{n_{y}} \Phi(x, y) d s(y)\right|_{ \pm} & =\int_{\Gamma} \delta(y) \partial_{n_{y}} \Phi(x, y) d s(y) \pm \frac{1}{2} \delta(x) .
\end{aligned}
$$


The jump conditions do not change, if we replace $\Phi$ by $\Phi_{0}$ on both sides. Using

$$
\begin{aligned}
\left(\operatorname{div} H^{E}\right)(x) & =\Delta \int_{\Gamma} \lambda(y) \Phi(x, y) d s(y)+\int_{\Gamma} \operatorname{div}_{x}(n(y) \delta(y) \Phi(x, y)) d s(y) \\
& =-k^{2} \int_{\Gamma} \lambda(y) \Phi(x, y) d s(y)+\int_{\Gamma} \delta(y) n(y) \cdot \operatorname{grad}_{x} \Phi(x, y) d s(y) \\
& =-k^{2} \int_{\Gamma} \lambda(y) \Phi(x, y) d s(y)-\int_{\Gamma} \delta(y) \partial_{n_{y}} \Phi(x, y) d s(y),
\end{aligned}
$$

$\mathrm{x} \in \mathrm{G}^{\mathrm{E}}$,

we deduce

$$
\begin{aligned}
H_{+}^{L}(x) & =\int_{\Gamma} \lambda(y) \operatorname{grad}_{x} \Phi_{0}(x, y) d s(y)-\frac{1}{2} n(x) \lambda(x), \\
H_{-}^{E}(x) & =\int_{\Gamma} \operatorname{curl}_{x}(a(y) \Phi(x, y)) d s(y)+\frac{1}{2} n(x) \wedge a(x) \\
& +\int_{\Gamma} \lambda(y) \operatorname{grad}_{x} \Phi(x, y) d s(y)+\frac{1}{2} n(x) \lambda(x) \\
& +\int_{\Gamma} n(y) \delta(y) \Phi(x, y) d s(y), \\
\left(\operatorname{div} H^{E}\right)_{-}(x)=-k^{2} \int_{\Gamma} \lambda(y) \Phi(x, y) d s(y) & -\int_{\Gamma} \delta(y) \partial_{n_{y}} \Phi(x, y) d s(y)+\frac{1}{2} \delta(x) .
\end{aligned}
$$

Introducing the operators

$$
\begin{aligned}
& (M a)(x)=2 n(x) \wedge \int_{\Gamma} \operatorname{curl}_{x}(a(y) \Phi(x, y)) d s(y) \\
& (N a)(x)=2 n(x) \cdot \int_{\Gamma} \operatorname{curl}_{x}(a(y) \Phi(x, y)) d s(y) \\
& (K \lambda)(x)=2 \int_{\Gamma} \lambda(y) \partial_{n_{y}} \Phi(x, y) d s(y) \\
& \left(K^{\prime} \lambda\right)(x)=2 \int_{\Gamma} \lambda(y) \partial_{n_{x}} \Phi(x, y) d s(y) \\
& (S \lambda)(x)=2 \int_{\Gamma} \lambda(y) \Phi(x, y) d s(y) \\
& (P \lambda)(x)=2 n(x) \wedge \int_{\Gamma} n(y) \lambda(y) \Phi(x, y) d s(y) \\
& (Q \lambda)(x)=2 n(x) \cdot \int_{\Gamma} n(y) \lambda(y) \Phi(x, y) d s(y) \\
& (R \lambda)(x)=2 n(x) \wedge \int_{\Gamma} \lambda(y) \operatorname{grad} x \Phi(x, y) d s(y)
\end{aligned}
$$




$$
\begin{aligned}
& \left(K_{0}^{\prime} \lambda\right)(x)=2 \int_{\Gamma} \lambda(y) \partial_{n_{x}} \Phi_{0}(x, y) d s(y), \\
& \left(R_{0} \lambda\right)(x)=2 n(x) \wedge \int_{\Gamma} \lambda(y) \operatorname{grad}_{x} \Phi_{0}(x, y) d s(y),
\end{aligned}
$$

we get the following expressions for the boundary values of $\mathrm{H}^{\mathrm{L}}$ and $\mathrm{H}^{\mathrm{E}}$ :

$$
\begin{aligned}
2 n(x) \wedge H_{+}^{L}(x) & =\left(R_{0} \lambda\right)(x), \\
2 n(x) \wedge H_{-}^{E}(x) & =(M a)(x)-a(x)+(R \lambda)(x)+(P \delta)(x), \\
2 n(x) \cdot\left(\mu^{L} H_{+}^{L}(x)\right) & =\mu^{L}\left(\left(K_{0}^{\prime} \lambda\right)(x)-\lambda(x)\right), \\
2 n(x) \cdot\left(\mu^{E} H_{-}^{E}(x)\right) & =\mu^{E}\left((N a)(x)+\left(K^{\prime} \lambda\right)(x)+\lambda(x)+(Q \delta)(x)\right), \\
2\left(\operatorname{div} H^{E}\right)(x) & =-k^{2}(S \lambda)(x)+\delta(x)-(K \delta)(x) .
\end{aligned}
$$

Thus, we immediately see that solving $(10)$ is equivalent to solving the integral equation

$$
A v=b, \quad A=\left(\begin{array}{ccc}
M-I & R-R_{0} & P \\
\mu^{E} N & \mu^{E}\left(I+K^{\prime}\right)+\mu^{L}\left(I-K_{0}^{\prime}\right) & \mu^{E} Q \\
0 & -k^{2} S & I-K
\end{array}\right), \quad v=\left(\begin{array}{l}
a \\
\lambda \\
\delta
\end{array}\right), \quad b=2\left(\begin{array}{l}
c \\
g \\
d
\end{array}\right) .
$$

\section{The integral equation (12) is of second kind in $X_{d}^{0 \alpha}(\Gamma)$}

According to $[1,2,3,9]$ the operators defined above have the following mapping properties

$$
\begin{array}{ll}
M: T^{0 \alpha}(\Gamma) \rightarrow T^{0 \alpha}(\Gamma) \text { resp. } T_{d}^{0 \alpha}(\Gamma) \rightarrow T_{d}^{0 \alpha}(\Gamma), & N: T_{d}^{0 \alpha}(\Gamma) \rightarrow C^{0 \alpha}(\Gamma), \\
Q, S, K, K^{\prime}, K_{0}^{\prime}: C^{0 \alpha}(\Gamma) \rightarrow C^{0 \alpha}(\Gamma), & P: C^{0 \alpha}(\Gamma) \rightarrow T_{d}^{0 \alpha}(\Gamma), \\
R, R_{0}: C^{0 \alpha}(\Gamma) \rightarrow T^{0 \alpha}(\Gamma) . &
\end{array}
$$

$\mathrm{N}, \mathrm{R}, \mathrm{R}_{0}$ are continuous, $\mathrm{M}, \mathrm{Q}, \mathrm{S}, \mathrm{K}, \mathrm{K}^{\prime}, \mathrm{K}_{0}^{\prime}, \mathrm{P}, \mathrm{R}-\mathrm{R}_{0}$ are compact.

Setting

$$
F(x)=2 \int_{\Gamma} \lambda(y) \operatorname{grad}_{x} \Phi(x, y) d s(y), \quad \lambda \in C^{0 \alpha}(\Gamma), \quad x \in G^{E},
$$

we get

$$
\begin{aligned}
& F \in C^{2}\left(G^{E}\right) \cap C^{0 \alpha}\left(\bar{G}^{E}\right), \\
& \operatorname{curl} F=0 \quad \text { in } G^{E}, \\
& \left.n \wedge F\right|_{\Gamma}=R \lambda .
\end{aligned}
$$

According to [1] we deduce

$\operatorname{Div}(R \lambda)=\operatorname{Div}(n \wedge F)=-\left.n \cdot \operatorname{curl} F\right|_{\Gamma}=0$ 
and therefore

$$
\|R \lambda\|_{d \alpha, \Gamma}=\|R \lambda\|_{T \alpha \cdot \Gamma} .
$$

In the same way we show $\left\|R_{0} \lambda\right\|_{d \alpha, \Gamma}=\left\|R_{0} \lambda\right\|_{T \alpha, \Gamma}$, so that the continuity of $R, R_{0}$ and the compactness of $R-R_{0}$ carry over to the case where we consider $R, R_{0}$ as operators mapping $C^{0 \alpha}(\Gamma)$ into $T_{d}^{0 \alpha}(\Gamma)$.

Now $A$ is split up into

$$
A=B+C, \quad B=\left(\begin{array}{ccc}
-1 & 0 & 0 \\
\mu^{E} N & \left(\mu^{E}+\mu^{L}\right) 1 & 0 \\
0 & 0 & 1
\end{array}\right), \quad C=\left(\begin{array}{ccc}
M & R-R_{0} & P \\
0 & \mu^{E} K^{\prime}-\mu^{L} K_{0}^{\prime} & \mu^{E} Q \\
0 & -k^{2} S & -K
\end{array}\right) .
$$

From the above considerations we immediately get

$$
B, C: X_{d}^{0 \alpha}(\Gamma) \rightarrow X_{d}^{0 \alpha}(\Gamma)
$$

where B is continuously invertible and C is compact. Therefore (12) is of second kind.

\section{The operator $A$ is injective}

Consider a solution $v=\left(\begin{array}{l}a \\ \lambda \\ \delta\end{array}\right) \in X_{d}^{0 \alpha}(\Gamma)$ of the homogeneous equation $A v=0$. Inserting $a, \lambda, \delta$ in (11), the fields $\mathrm{H}^{\mathrm{L}}$ and $\mathrm{H}^{\mathrm{E}}$ obtained in this way, solve the homogeneous problem (10). Corresponding to Theorem 2, they vanish identically. But $\mathrm{H}^{\mathrm{L}}$ was defined as

$$
H^{L}(x)=\operatorname{grad}_{x} \int_{\Gamma} \lambda(y) \Phi_{0}(x, y) d s(y)
$$

and therefore

$$
0=2 \mathrm{n} \cdot \mathrm{H}_{+}^{\mathrm{L}}=2 \int_{\Gamma} \lambda \partial_{\mathrm{n}_{\mathbf{x}}} \Phi_{0} \mathrm{ds}-\lambda=-\left(\mathrm{I}-\mathrm{K}_{0}^{\prime}\right) \lambda .
$$

Since $G^{L}=\mathbb{R}^{3} \backslash \bar{G}^{E}$ is connected and unbounded, we get $N\left(I-K_{0}^{\prime}\right)=\{0\}$ [4], so

$$
\lambda=0 \text {. }
$$

Using $\lambda=0$ we obtain from the last component of $A v=b$

$$
(I-K) \delta=0 \text {. }
$$

According to [1], N(I-K) $=\{0\}$ for $\operatorname{Im}(k)>0$ and thus

$$
\delta=0 \text {. }
$$

From the first component of $A v=b$ we get

$$
(I-M) a=0
$$

and again

$$
a=0
$$

because $N(I-M)=\{0\}$ for $\operatorname{Im}(k)>0[1]$. 
From the above considerations we conclude that our auxiliary problem $(10)$ is equivalent to the integral equation $A v=b$. Since $c \in T_{d}^{0 \alpha}(\Gamma), g \in C^{0 \alpha}(\Gamma), d \in C^{0 \alpha}(\Gamma)$, the right hand side $b$ lies in $X_{d}^{0 \alpha}(\Gamma)$. Now $A$ is compact and injective in $X_{d}^{0 \alpha}(\Gamma)$ and therefore, according to the Riesz-theory, continuously invertible in $X_{d}^{0 \alpha}(\Gamma)$. So $A v=b$ is solvable for any $b \in X_{d}^{0 \alpha}(\Gamma)$, with $v$ depending continuously on $b$. If we use the components of $v$ to define $\mathrm{H}^{\mathrm{L}}, \mathrm{H}^{\mathrm{E}}$ using (11), we get a solution of (10). By

$$
\begin{aligned}
& \left\|H^{L}\right\|_{V \alpha, \bar{G}^{L}} \leq c_{\alpha}\|\lambda\|_{0 \alpha, \Gamma}, \\
& \max \left(\left\|H^{E}\right\|_{V \alpha, \bar{G}^{E}},\left\|\operatorname{div} H^{E}\right\|_{0 \alpha, \dot{G}^{E}}\right) \leq c_{\alpha}\left\|\left(\begin{array}{l}
a \\
\lambda \\
\delta
\end{array}\right)\right\|_{X_{d} \alpha} \text {, } \\
& \left\|\operatorname{curl} \mathrm{H}^{\mathrm{E}}\right\|_{\mathrm{V} \alpha, \overline{\mathrm{G}}^{\mathrm{E}}} \leq \mathrm{c}_{\alpha} \max \left(\|\mathrm{a}\|_{\mathrm{d} \alpha, \Gamma},\|\delta\|_{0 \alpha, \Gamma}\right) \text {, }
\end{aligned}
$$

we get the continuous dependence of $\mathrm{H}^{\mathrm{L}}$ and $\mathrm{H}^{\mathrm{E}}$ on the data $c, g$, $\mathrm{d}$.

Up to now, we have shown the unique solvability of the auxialiary problem (10) for arbitrary $c \in T_{d}^{0 \alpha}(\Gamma), g \in C^{0 \alpha}(\Gamma), d \in C^{0 \alpha}(\Gamma)$. With the help of this result, we want to prove existence and uniqueness for (3), (4), (5) under the additional assumption $(6)$ of prescribed circulations for $\mathrm{H}^{\mathrm{L}}$.

\section{Lemma 2}

(i) Let $G^{J} \subset G^{L}$ be defined as above, $\rho \in C^{0 \alpha}\left(\mathbb{R}^{3}\right)$, supp $(\rho) \subset G^{J}$. Then

$$
\begin{aligned}
& u(x)=\int_{G^{J}} \rho(y) \Phi_{0}(x, y) d y \in C^{2}\left(\mathbb{R}^{3}\right), \\
& u(x)=O\left(\frac{1}{|x|}\right), \quad \operatorname{grad} u(x)=O\left(\frac{1}{|x|}\right), \quad|x| \rightarrow \infty .
\end{aligned}
$$

(ii) Consider $J_{e} \in C^{\prime}\left(\mathbb{R}^{3}\right), \operatorname{div} J_{e}=0, \quad \operatorname{supp}\left(J_{e}\right) \subset G^{J}$. There exist

$$
\begin{aligned}
& H^{J} \in C^{1}\left(\mathbb{R}^{3}\right), \quad \quad E^{J} \in C^{1}\left(G^{L}\right) \cap C\left(\bar{G}^{L}\right) \text {, } \\
& \operatorname{curl} \mathbf{H}^{J}=J_{e}, \quad \operatorname{div} H^{J}=0, \quad H^{J}(x)=O\left(\frac{1}{|x|^{2}}\right), \quad \text { uniformly for }|x| \rightarrow \infty \text {, } \\
& \text { curl } E^{J}=i \omega \mu^{L} H^{J}, \quad \operatorname{div} E^{J}=0, \quad E^{J}(x)=O\left(\frac{1}{|x|}\right), \quad \text { uniformly for }|x| \rightarrow \infty .
\end{aligned}
$$

(iii) Corresponding to the Neumann fields in $G^{L}$ we have $E_{i}^{Z} \in C^{1}\left(G^{L}\right) \cap C\left(\bar{G}^{L}\right)$, curl $E_{i}^{Z}=i \omega \mu^{L} Z_{i}^{L}, \quad \operatorname{div} E_{i}^{Z}=0, \quad E_{i}^{Z}(x)=O\left(\frac{1}{|x|}\right), \quad$ uniformly for $\quad|x| \rightarrow \infty$. 


\section{Proof}

The first part is an easy consequence of some well known properties of the Newtonian potentials in $\mathbb{R}^{3}[5]$.

In (ii), we assume $J_{e} \in C^{1}\left(\mathbb{R}^{3}\right), \operatorname{supp}\left(J_{e}\right) \subset G^{J}$. Therefore $J_{e} \in C^{0 \alpha}\left(\mathbb{R}^{3}\right)$ and from (i) we get

$$
A=J_{e} * \Phi_{0}=\int_{G^{J}} J_{e}(y) \Phi_{0}(x, y) d y \in C^{2}\left(\mathbb{R}^{3}\right) \text {. }
$$

Defining $H^{J}$ as $H^{J}=\operatorname{curl} A \in C^{1}\left(\mathbb{R}^{3}\right)$, we see that

$$
\operatorname{div} \mathbf{H}^{\mathrm{J}}=0, \quad \operatorname{curl} \mathbf{H}^{\mathrm{J}}=\operatorname{curl} \operatorname{curl} \mathrm{A}=(\operatorname{grad} \operatorname{div}-\Delta) A=\mathrm{J}_{\mathrm{e}} .
$$

Corresponding to (i), the components of $H^{J}$ behave as $O\left(\frac{1}{|x|^{2}}\right)$ uniformly for $|x| \rightarrow \infty$. Since

$$
\int_{\Gamma_{j}} n \cdot H^{J} d s=\int_{\Gamma_{j}} n \cdot \operatorname{curl} A d s=0, \quad j=1, \ldots, m,
$$

we may apply Lemma 1 to $H^{J}$ (with $\beta=1$ ) and get the existence of $E^{J}$.

For the last part, we remark, that $Z_{i}^{L} \in C^{1}\left(G^{L}\right) \cap C\left(\bar{G}^{L}\right), i=1, \ldots, p$, are harmonic vector fields in $G^{\mathbf{L}}$ satisfying

$$
n \cdot Z_{i}^{L}=0 \quad \text { on } \Gamma, \quad Z_{i}^{L}=O\left(\frac{1}{|x|^{2}}\right), \quad \text { uniformly for } \quad|x| \rightarrow \infty .
$$

Using again Lemma 1 with $\beta=1$, the proof is completed.

With the help of Lemma 2, we obtain the main result of this paper.

\section{Theorem 4}

Consider $J_{e} \in C^{1}\left(\mathbb{R}^{3}\right), \operatorname{div} J_{e}=0, \operatorname{supp}\left(J_{e}\right) \subset G^{J}, \bar{G}^{J} \subset G^{L}, G^{J}$ bounded. Under these assumptions, problem $(3),(4),(5),(6)$ possesses a solution $H^{L}, E^{L}, H^{E}, E^{E}$. $\mathrm{H}^{\mathrm{L}}, \mathrm{H}^{\mathrm{E}}, \mathrm{E}^{\mathrm{E}}$ are uniquely determined.

\section{Proof} and

Consider $H^{J}$, which is given by Lemma 2 . Define $h_{i}^{J}$ as $h_{i}^{J}=\int_{\gamma_{i}^{L}} \tau \cdot H^{J} d l, i=1, \ldots, p$,

$$
H^{Z}=\sum_{j=1}^{p}\left(h_{j}^{L}-h_{j}^{J}\right) Z_{j}^{L},
$$




$$
\begin{aligned}
& c=\left.n \wedge\left(H^{J}+H^{Z}\right)\right|_{\Gamma}, \\
& g=\left.n \cdot\left(\mu^{L}\left(H^{J}+H^{Z}\right)\right)\right|_{\Gamma}=\left.n \cdot\left(\mu^{L} H^{J}\right)\right|_{\Gamma} .
\end{aligned}
$$

From $H^{J} \in C^{1}\left(R^{3}\right)$ we deduce

$$
\left.H^{J}\right|_{\Gamma} \in C^{0 \alpha}(\Gamma)
$$

For the surface divergence of $n \wedge H^{J}$ on $\Gamma$ we get according to [1]

$\operatorname{Div}\left(n \wedge H^{J}\right)=-\left.n \cdot \operatorname{curl} H^{J}\right|_{\Gamma}=-\left.n \cdot J_{e}\right|_{\Gamma}=0$,

because $\operatorname{supp}\left(J_{e}\right) \subset G^{J}, \bar{G}^{J} \subset G^{L}$.

For the Neumman fields $Z_{\mathbf{i}}^{\mathbf{L}}$ holds [6]

$$
\left.\mathrm{n} \wedge \mathrm{Z}_{\mathrm{i}}^{\mathrm{L}}\right|_{\Gamma} \in \mathrm{C}^{0 \alpha}(\Gamma), \quad \operatorname{Div}\left(\mathrm{n} \wedge \mathrm{Z}_{\mathrm{i}}^{\mathrm{L}}\right)=-\left.\mathrm{n} \cdot \operatorname{curl} \mathrm{Z}_{\mathrm{i}}^{\mathrm{L}}\right|_{\Gamma}=0 .
$$

and therefore

$$
c \in \mathrm{T}_{\mathrm{d}}^{0 \alpha}(\Gamma)
$$

On the other hand $H^{J} \in C^{1}\left(\mathbb{R}^{3}\right)$ implies

$$
g \in C^{0 \alpha}(\Gamma)
$$

By Theorem 3 there exist unique fields $\tilde{H}^{L}, \tilde{H}^{E}$,

$$
\begin{aligned}
& \tilde{H}^{L} \in C^{1}\left(G^{L}\right) \cap C\left(\bar{G}^{L}\right), \\
& \tilde{H}^{E} \in C^{2}\left(G^{E}\right) \cap C\left(\bar{G}^{E}\right), \quad \operatorname{div} \tilde{H}^{E} \in C\left(\bar{G}^{E}\right), \quad \operatorname{curl} \tilde{H}^{E} \in C\left(\bar{G}^{E}\right)
\end{aligned}
$$

solving

$$
\begin{aligned}
& \operatorname{curl} \tilde{\mathrm{H}}^{\mathrm{L}}=0 \\
& \operatorname{div} \tilde{H}^{\mathrm{L}}=0 \\
& \left(\Delta+\mathrm{k}^{2}\right) \tilde{\mathrm{H}}^{\mathrm{E}}=0 \\
& k^{2}=i \omega \sigma^{E}{ }^{E} \\
& \text { in } \mathrm{G}^{\mathrm{E}} \text {, } \\
& \mathrm{n} \wedge \tilde{\mathrm{H}}^{\mathrm{E}}-\mathrm{n} \wedge \tilde{\mathrm{H}}^{\mathbf{L}}=\mathrm{c} \\
& n \cdot\left(\mu^{E} \tilde{H}^{E}\right)-n \cdot\left(\mu^{L} \tilde{H}^{L}\right)=g \\
& \text { on } \Gamma \text {, } \\
& \operatorname{div} \tilde{\mathrm{H}}^{\mathrm{E}}=0 \\
& \int_{\gamma_{i}^{L}} \tau \cdot \tilde{\mathbf{H}}^{L} \mathrm{dl}=0 \\
& i=1, \ldots, p, \\
& \tilde{H}^{L}(x)=o(1) \\
& \text { uniformly for }|x| \rightarrow \infty \text {. }
\end{aligned}
$$

From $\operatorname{div} \tilde{\mathrm{H}}^{\mathrm{E}}=0$ on $\Gamma$ and $\operatorname{Im}(\mathrm{k})>0$ we get in the same way as in the proof of Theorem 2, that $\tilde{\mathrm{H}}^{\mathrm{E}}$ and $\tilde{\mathrm{E}}^{\mathrm{E}}=\frac{1}{\sigma^{E}}$ curl $\tilde{\mathrm{H}}^{\mathrm{E}}$ solve the time-harmonic Maxwell equations in $G^{E}$ with coefficients $\mu^{E}, \sigma^{E}$ and $\omega$. 
$\tilde{H}^{L}$ is harmonic in $G^{L}$. From the proof of Theorem 3 , we know, that for $\tilde{H}^{L}$ even the stronger condition $\tilde{H}^{L}(x)=O\left(\frac{1}{|x|^{2}}\right)$ uniformly for $|x| \rightarrow \infty$ holds. In addition we have

$$
\begin{aligned}
i \omega \mu^{L} \int_{\Gamma_{j}} n \cdot \tilde{H}^{L} d s & =i \omega \mu^{E} \int_{\Gamma_{j}} n \cdot \tilde{H}^{E} d s-i \omega \int_{\Gamma_{j}} g d s \\
& =\int_{\Gamma_{j}} n \cdot \operatorname{curl} \tilde{E}^{E} d s-i \omega \mu^{L} \int_{\Gamma_{j}} n \cdot H^{J} d s \\
& =\int_{\Gamma_{j}} n \cdot \operatorname{curl} \tilde{E}^{E} d s-i \omega \mu^{L} \int_{\Gamma_{j}} n \cdot \operatorname{curl} A d s, \quad j=1, \ldots, m,
\end{aligned}
$$

A being the convolution $A=J_{e} * \Phi_{0}$ from the proof of Lemma 2 . Since $\Gamma_{j}, j=1, \ldots, m$ are closed surfaces, we conclude by Stokes' theorem

$$
\int_{\Gamma_{j}} n \cdot \tilde{H}^{L} d s=0, \quad j=1, \ldots, m .
$$

Now Lemma 1 guarantees the existence of $\widetilde{E}^{L} \in C^{1}\left(G^{L}\right) \cap C\left(\bar{G}^{L}\right)$ with $\operatorname{curl} \tilde{E}^{L}=i \omega \mu^{L} \tilde{H}^{L} \quad$ in $G^{L}, \quad \tilde{E}^{L}(x)=O\left(\frac{1}{|x|}\right), \quad$ uniformly for $|x| \rightarrow \infty$.

Summarizing the results obtained for $\tilde{\mathrm{H}}^{\mathbf{L}}, \tilde{\mathrm{E}}^{\mathbf{L}}, \tilde{\mathrm{H}}^{\mathrm{E}}, \tilde{\mathrm{E}}^{\mathrm{E}}$, we have

$$
\begin{aligned}
& \tilde{\mathrm{II}}^{\mathrm{L}}, \tilde{\mathrm{E}}^{\mathrm{L}} \in \mathrm{C}^{1}\left(\mathrm{G}^{\mathrm{L}}\right) \cap \mathrm{C}\left(\overline{\mathrm{G}}^{\mathrm{L}}\right), \quad \tilde{\mathrm{H}}^{\mathrm{E}}, \tilde{\mathrm{E}}^{\mathrm{E}} \in \mathrm{C}^{1}\left(\mathrm{G}^{\mathrm{E}}\right) \cap \mathrm{C}\left(\overline{\mathrm{G}}^{\mathrm{E}}\right) \\
& \begin{array}{lll}
\operatorname{curl} \tilde{\mathrm{H}}^{\mathrm{L}}=0 & \text { in } \mathrm{G}^{\mathrm{L}}, & \operatorname{curl} \tilde{\mathrm{H}}^{\mathrm{E}}=\sigma^{\mathrm{E}} \tilde{\mathrm{E}}^{\mathrm{E}} \\
\operatorname{curl} \tilde{\mathrm{E}}^{\mathrm{L}}=i \omega \mu^{\mathrm{L}} \tilde{\mathrm{H}}^{\mathrm{L}} & & \operatorname{curl} \tilde{\mathrm{E}}^{\mathrm{E}}=i \omega \mu^{\mathrm{E}} \tilde{\mathrm{H}}^{\mathrm{E}}
\end{array} \quad \text { in } G^{E}, \\
& \begin{array}{cc}
\mathrm{n} \wedge \tilde{\mathrm{H}}^{\mathrm{E}}-\mathrm{n} \wedge \tilde{\mathrm{H}}^{\mathrm{L}}=\mathrm{c} & \\
\mathrm{n} \cdot\left(\mu^{\mathrm{E}} \tilde{\mathrm{H}}^{\mathrm{E}}\right)-\mathrm{n} \cdot\left(\mu^{\mathrm{L}} \tilde{\mathrm{H}}^{\mathrm{L}}\right)=\mathrm{g} & \text { on } \Gamma, \\
\int_{\mathrm{r}_{\mathrm{i}}} \tau \cdot \tilde{\mathrm{H}}^{\mathrm{L}} \mathrm{dl}=0 & \mathrm{i}=1, \ldots, \mathrm{p},
\end{array} \\
& \tilde{H}^{L}(x)=o(1), \quad \tilde{E}^{L}(x)=o(1) \quad \text { uniformly for }|x| \rightarrow \infty \text {. }
\end{aligned}
$$

But in Lemma 2 the existence of $E^{J}, E^{Z} \in C^{1}\left(G^{l}\right) \cap C\left(\bar{G}^{L}\right)$,

$$
\operatorname{curl} E^{J}=i \omega \mu^{L} H^{J}, \quad \quad \operatorname{curl} E^{Z}=i \omega \mu^{L} H^{Z},
$$

is shown, both behaving uniformly like o(1) for $|x| \rightarrow \infty$.

Defining $\mathrm{H}^{\mathrm{L}}, \mathrm{E}^{\mathrm{L}}, \mathrm{H}^{\mathrm{E}}, \mathrm{E}^{\mathrm{E}}$ as

$$
H^{L}=\widetilde{H}^{L}+H^{J}+H^{Z}, \quad E^{L}=\widetilde{E}^{L}+E^{J}+E^{Z}, \quad H^{E}=\widetilde{H}^{E}, \quad E^{E}=\tilde{E}^{E},
$$


and using curl $\mathrm{H}^{\mathrm{J}}=\mathrm{J}_{\mathrm{e}}$, curl $\mathrm{H}^{\mathrm{Z}}=0$, we get

$$
\begin{array}{rlr}
\operatorname{curl} H^{L} & =\operatorname{curl}\left(\tilde{H}^{L}+H^{J}+H^{Z}\right)=J_{e} & \\
\operatorname{curl} E^{L} & =\operatorname{curl}\left(\tilde{E}^{L}+E^{J}+E^{Z}\right)=i \omega \mu^{L} H^{L} & \text { in } G^{L}, \\
\operatorname{curl} H^{E} & =\sigma^{E} E^{E} & \\
\operatorname{curl} E^{E} & =i \omega \mu^{E} H^{E} & \text { in } G^{E}, \\
n \wedge H^{E} & =n \wedge \tilde{H}^{E}=n \wedge \tilde{H}^{L}+c=n \wedge\left(\tilde{H}^{L}+H^{J}+H^{Z}\right)=n \wedge H^{L} & \\
n \cdot\left(\mu^{E} H^{E}\right) & =n \cdot\left(\mu^{E} \cdot \tilde{H}^{E}\right)=n \cdot\left(\mu^{L} \tilde{H}^{L}\right)+g=n \cdot\left(\mu^{L}\left(\tilde{H}^{L}+H^{J}+H^{Z}\right)\right) & \text { on } \Gamma \\
& =n \cdot\left(\mu^{L} H^{L}\right) &
\end{array}
$$

and

$$
\int_{\gamma_{i}^{L}} \tau \cdot H^{L} d l=\int_{\gamma_{i}^{L}} \tau \cdot\left(\tilde{H}^{L}+H^{J}+H^{Z}\right) d l=\int_{\gamma_{i}^{L}} \tau \cdot H^{J} d l+h_{i}^{L}-h_{i}^{J}=h_{i}^{L}, \quad i=1, \ldots, p .
$$

Therefore $\mathrm{H}^{\mathrm{L}}, \mathrm{E}^{\mathrm{L}}, \mathrm{H}^{\mathrm{E}}, \mathrm{E}^{\mathrm{E}}$ solve $(3),(4),(5),(6)$.

\section{Corollary}

For $J_{e} \in C^{l}\left(\mathbb{R}^{3}\right), \operatorname{div} J_{e}=0, \operatorname{supp}\left(J_{e}\right) \subset G^{J}, \bar{G}^{J} \subset G^{L}$ bounded, problem (3), (4), (5) is solvable.

In the homogeneous case $J_{e}=0$ we get exactly $p$ linear independent solutions $\mathrm{H}^{\mathrm{L}}, \mathrm{H}^{\mathrm{E}}, \mathrm{E}^{\mathrm{E}}$, where $\mathrm{p}$ denotes the topological genus of $\mathrm{G}^{\mathrm{E}}$ resp. $\mathrm{G}^{\mathrm{L}}$.

$\mathrm{E}^{\mathbf{L}}$ is not uniquely determined.

\section{Proof}

The first statement follows immediately from the last theorem by chosing the circulations $h_{i}^{L}, i=1, \ldots, p$ arbitrarily.

In the case $J_{e}=0$ Theorem 3 shows the existence of $p$ solutions $H_{j}^{L}, E_{j}^{L}, H_{j}^{E}, E_{j}^{E}$, $j=1, \ldots, p$, of $(3),(4),(5)$, having circulations $h_{j i}^{L}=\delta_{i j}, i=1, \ldots, p$. The linear independence of $H_{j}^{L}, E_{j}^{L}, H_{j}^{E}$ is a consequence of the uniqueness results of Theorem 1 .

The nonuniqueness of $E^{L}$ is obvious. 


\section{References}

[1] Colton D. Kress R. "Integral equation methods in scattering theory". J. Wiley. 1983

[2] Knauff W. Kress R. "On the exterior boundary - value problem for the time-harmonic Maxwell equations". J. of math. An. and App. 72, 1979

[3] Kress R. "On the boundary operator in electromagnetic scattering". Proc. R. Soc. Edinburgh 103 A. 1986

[4] Kress R. "Linear Inlegral Equations". Springer 1989

[5] L'ons J. L., Dautray R. "Mathematical Analysis and Numerical Methods for Science and Technology". Vol. 1. Springer 1990

[6] Martensen E., "Potentialtheorie". Teubner 1968

[7] Müller C.. "Grundprobleme der mathematischen Theorie elektromagnetischer Schwingungen". Springer 1957

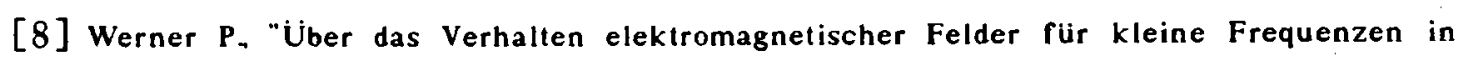
mehrfach zusammenhängenden Gebieten":

J. für Reine und Angewandte Math. 1.278/79,1975 resp. 2,280,1976

[9] Wilde P., "Über Transmissionsprobleme bei der vektoriellen Helmholtzgleichung". PhD Thesis. Göttingen 1985 